\title{
Fuzzy reproducing kernel space method for solving fuzzy boundary value problems
}

\author{
N. Gholami ${ }^{1} \cdot$ T. Allahviranloo ${ }^{2}$ [D $\cdot$ S. Abbasbandy ${ }^{3} \cdot$ N. Karamikabir $^{1}$
}

Received: 12 November 2018 / Accepted: 16 March 2019 / Published online: 29 March 2019

(c) The Author(s) 2019

\begin{abstract}
In the beginning, we describe the fuzzy inner product space and the fuzzy Hilbert space. Our goal is to use the fuzzy reproducing kernel method to solve the second-order fuzzy boundary value problem. The fuzzy convergence analysis of introduced method is discussed in detail. We present some examples in the end.
\end{abstract}

Keywords Fuzzy Hilbert space $\cdot$ Fuzzy convergence $\cdot$ Fuzzy reproducing kernel method

\section{Introduction}

Reproducing kernel method is one of the most basic methods for approximation. In doing so, our main goal is to solve fuzzy boundary value problems using fuzzy reproducing kernel methods. We discuss the convergence of our procedure using the concept of fuzzy distance. At the end, there are some examples. We describe the fuzzy reproducing kernel method (FRKM for short) for solving the following fuzzy boundary value problem:

$\left\{\begin{array}{l}y^{\prime \prime}(x)+m(x) y^{\prime}(x)+n(x) y(x)=f(x) \quad 0<x<1, \\ y(0)=0, \quad y(1)=0\end{array}\right.$

here, $f(x)$ is a fuzzy function. The functions of $m(x)$ and $n(x)$ are ordinary and continuous. The process of doing this paper is as follows: In the part of preliminaries, the fundamental concepts that have been used in later sections are presented. In "Fuzzy reproducing kernel space" section, we have defined the fuzzy reproducing kernel (FRK for short). Fuzzy inner product spaces and fuzzy reproducing kernel spaces have been introduced. Also, the solution to Eq. (1.1) is given

T. Allahviranloo

allahviranloo@yahoo.com

1 Department of Mathematics, Hamedan Branch, Islamic Azad University, Hamedan, Iran

2 Faculty of Engineering and Natural Sciences, Bahcesehir University, Istanbul, Turkey

3 Department of Mathematics, Faculty of Science, Imam Khomeini International University, Qazvin, Iran with the initial boundary value conditions. In "Fuzzy convergence analysis" section, we bring the fuzzy convergence theorem. In the next section, we give some examples for a better understanding. At the end of this work, we bring the results. For more information on the reproducing kernel method and fuzzy convergence, see [1-3, 11].

\section{Preliminaries}

We introduce the primary definitions of the generalized Hukuhara difference, generalized Hukuhara derivative, Hausdorff distance and fuzzy continuous function. For definition of the fuzzy number and $\alpha$-level set see [10].

Remark 2.1 We assume that in the whole of this paper the generalized Hukuhara difference exists. We denote generalized Hukuhara difference by $\ominus_{\mathrm{gH}}$.

Definition 2.2 [5] Let $x$ and $y$ be two fuzzy numbers in $\mathbb{R}_{F}$, where $\mathbb{R}_{F}$ is the set of all fuzzy numbers. If the exists a fuzzy number as $z$ that satisfies in follows condition, then the generalized Hukuhara difference ( $\mathrm{gH}$-difference for short) is defined as

$x \ominus_{\mathrm{gH}} y=z \Leftrightarrow\left\{\begin{array}{c}\text { (i) } x=y+z \\ \text { or }(\text { ii) } y=x+(-1) z .\end{array}\right.$

where (i) and (ii) are both valid if and only if $z$ is a crisp number. 
Definition 2.3 [4] Let $x, y, z, w$ are fuzzy numbers and $\beta \in \mathbb{R}$. Define the Hausdorff distance as in [9]:

$D: \mathbb{R}_{F} \times \mathbb{R}_{F} \rightarrow \mathbb{R}^{+} \cup\{0\}$

$D(x, y)=\sup _{\alpha \in[0,1]} \max \left\{\left|x^{-}(\alpha)-y^{-}(\alpha) \| x^{+}(\alpha)-y^{+}(\alpha)\right|\right\}$.

By [13], we have:

1. $D(x \oplus z, y \oplus z)=D(x, y)$,

2. $D(\lambda x, \lambda y)=|\lambda| D(x, y)$,

3. $D(x \oplus y, z \oplus w) \leq D(x, z)+D(y, w)$,

4. $\quad D(x \ominus y, z \ominus w) \leq D(x, z)+D(y, w)$, until $x \ominus y$ and $z \ominus w$ are available.

In the above features, $\ominus$ is the Hukuhara difference. It is equal to $z \ominus y=x$ iff $x \oplus y=z$.

In the following, we denote the Hausdorff distance by $D$.

Definition 2.4 [14] Let $f:(\alpha, \beta) \rightarrow \mathbb{R}_{F}$ be a fuzzy-valued function. In this case, the generalized Hukuhara derivative of $f$ at $x_{o} \in(\alpha, \beta)$ is defined as

$f_{\mathrm{gH}}^{\prime}\left(x_{0}\right)=\lim _{\varepsilon \rightarrow 0} \frac{f\left(x_{0}+\varepsilon\right) \ominus_{\mathrm{gH}} f\left(x_{0}\right)}{\varepsilon}$.

If $f_{\mathrm{gH}}^{\prime}\left(x_{0}\right) \in \mathbb{R}_{F}$ satisfying (2.3) exists, $f$ is called generalized Hukuhara differentiable ( $\mathrm{gH}$-differentiable for short) at $x_{0}$.

Definition 2.5 [7] Let $f:[\alpha, \beta] \rightarrow \mathbb{R}_{F}$ be a fuzzy-valued function. In this case, $f$ at $x_{0} \in[\alpha, \beta]$ is continuous if $\forall \varepsilon>0, \exists \delta>0: D\left(f(x), f\left(x_{0}\right)\right)<\varepsilon$, when $x \in[\alpha, \beta]$ and $\left|x-x_{0}\right|<\delta$. If $f$ is continuous for all $x_{0} \in[\alpha, \beta]$, then $f$ is a fuzzy continuous on interval of $[\alpha, \beta]$.

\section{Fuzzy reproducing kernel space}

We will introduce the FRKM to solve the fuzzy boundary value problems. Initially, we create the fuzzy reproducing kernel space (FRKS for short) $W^{m}[0,1]$, while each fuzzy function satisfies in the $y(0)=0$ and $y(1)=0$.

Definition 3.1 (Fuzzy absolutely continuous function) If $f(x)$ is a fuzzy function on $[\alpha, \beta]$, and $\left\{\left(\alpha_{i}, \beta_{i}\right)\right\}_{i=1}^{n}$ is a set of disjoint open intervals $\left(\alpha_{i}, \beta_{i}\right) \subset[\alpha, \beta]$.

If for all $\varepsilon$, there is a $\delta$, which has no relation with $n$, such that $\sum_{k=1}^{n} D\left(f\left(\beta_{k}\right), f\left(\alpha_{k}\right)\right)<\varepsilon$ for $\sum_{k=1}^{n}\left(\beta_{k}-\alpha_{k}\right)<\delta$, then $f(x)$ is a fuzzy absolutely continuous function (FACF for short) on $[a, b]$.

Definition 3.2 Define the fuzzy space $W^{m}[0,1]$ as $W^{m}[0,1]=\left\{y(x) \mid y\right.$ is a fuzzy function, $y_{\mathrm{gH}}^{(m-1)}(x)$ is a FACF, $y_{\mathrm{gH}}^{(m)}(x) \in L^{2}[0,1], L^{2}[0,1]$ is a fuzzy space, $y(0)=0, y(1)=0\}$.
We define the fuzzy inner product (FIP for short) in this space as

$\langle y, z\rangle_{m}=\sum_{k=0}^{m-1} y_{\mathrm{gH}}^{(k)}(0) \odot z_{\mathrm{gH}}^{(k)}(0) \oplus \int_{0}^{1} y_{\mathrm{gH}}^{(m)}(x) \odot$

$z_{\mathrm{gH}}^{(m)}(x) \mathrm{d} x ; \quad y, z \in W^{m}[0,1]$,

where $\langle y, z\rangle_{m}$ is the fuzzy inner product in the fuzzy space $W^{m}[0,1]$. Also, fuzzy norm in this space is defined as $\|y\|_{m}=\sqrt{\langle y, y\rangle_{m}}$.

Definition 3.3 Define the fuzzy space $W^{1}[0,1]$ as $W^{1}[0,1]=\{y(x) \mid y$ is a fuzzy function, $y(x)$ is a FACF, $y_{\mathrm{gH}}^{\prime}(x) \in L^{2}[0,1], L^{2}[0,1]$ is a fuzzy space $\}$. We define the FIP in this space as

$\langle y, z\rangle_{1}=y(0) \odot z(0) \oplus \int_{0}^{1} y_{\mathrm{gH}}^{\prime}(x) \odot z_{\mathrm{gH}}^{\prime}(x) \mathrm{d} x ; \quad y, z \in W^{1}[0,1]$,

where $\langle y, z\rangle_{1}$ is the fuzzy inner product in the fuzzy space $W^{1}[0,1]$.

Definition 3.4 Define the fuzzy space $L^{2}[0,1]$ as

$L^{2}[0,1]=\left\{y(x) \mid y\right.$ is a fuzzy function, $\left.\int_{0}^{1} y^{2}(x) \mathrm{d} x<\infty\right\}$.

We define the FIP in this space as

$\langle y, z\rangle_{L^{2}}=\int_{0}^{1} y(x) \odot z(x) \mathrm{d} x ; \quad y, z \in L^{2}[0,1]$,

where $\langle y, z\rangle_{L^{2}}$ is the fuzzy inner product in the fuzzy space $L^{2}[0,1]$.

Definition 3.5 If $f \in W^{m}[0,1]$, we define $\langle f, f\rangle_{m}=\|f\|_{m}^{2}=D^{2}(f, 0)$.

Using definition 3.1 in [8], we can deduce Lemmas 3.6 and 3.8 .

Lemma 3.6 Suppose that $F(\mathbb{R}, F(\mathbb{R}))$ is a vector space over $\mathbb{R}$. Also, the FIP in the fuzzy space $L^{2}[0,1]$ on $F(\mathbb{R}, F(\mathbb{R}))$ is a mapping as $\langle., .\rangle_{L^{2}}: F(\mathbb{R}, F(\mathbb{R})) \times F(\mathbb{R}, F(\mathbb{R})) \rightarrow F(\mathbb{R})$, with this property for each $k \in F(\mathbb{R})$ and all functions $f_{1}, f_{2}, f_{3} \in F(\mathbb{R}, F(\mathbb{R}))$, satisfies the following conditions:

1. $\left\langle f_{1}+f_{2}, f_{3}\right\rangle_{L^{2}}=\left\langle f_{1}, f_{3}\right\rangle_{L^{2}} \oplus\left\langle f_{2}, f_{3}\right\rangle_{L^{2}}$,

2. $\left\langle k f_{1}, f_{2}\right\rangle_{L^{2}}=k\left\langle f_{1}, f_{2}\right\rangle_{L^{2}}$ 
3. $\left\langle f_{1}, f_{2}\right\rangle_{L^{2}}=\left\langle f_{2}, f_{1}\right\rangle_{L^{2}}$,

4. $\left\langle f_{1}, f_{1}\right\rangle_{L^{2}}>0$,

5. if $f_{1} \neq 0$, then $\inf _{\alpha \in(0,1]}\left\langle f_{1}, f_{1}\right\rangle_{\alpha}^{-}>0$,

6. $\left\langle f_{1}, f_{1}\right\rangle_{L^{2}}=0$ iff $f_{1}=0$.

Proof To prove, we need to apply the fuzzy integral properties, the concept of the Hausdorff distance and the fuzzy norm.

1. $\left\langle f_{1} \oplus f_{2}, f_{3}\right\rangle_{L^{2}}=\int_{0}^{1}\left(\left(f_{1} \oplus f_{2}\right) \odot f_{3}\right)=$ $\int_{0}^{1}\left(f_{1} \odot f_{3}\right) \oplus \int_{0}^{1}\left(f_{2} \odot f_{3}\right)=\left\langle f_{1}, f_{3}\right\rangle_{L^{2}} \oplus\left\langle f_{2}, f_{3}\right\rangle_{L^{2}}$ $\left\langle f_{1} \oplus f_{2}, f_{3}\right\rangle_{L^{2}}=\int_{0}^{1}\left(\left(f_{1} \oplus f_{2}\right) \odot f_{3}\right)=$ $\int_{0}^{1}\left(f_{1} \odot f_{3}\right) \oplus \int_{0}^{1}\left(f_{2} \odot f_{3}\right)=\left\langle f_{1}, f_{3}\right\rangle_{L^{2}} \oplus\left\langle f_{2}, f_{3}\right\rangle_{L^{2}}$.

2. $\left\langle k \odot f_{1}, f_{2}\right\rangle_{L^{2}}=\int_{0}^{1}\left(k \odot f_{1} \odot f_{2}\right)=$ $k \odot \int_{0}^{1}\left(f_{1} \odot f_{2}\right)=k \odot\left\langle f_{1}, f_{2}\right\rangle_{L^{2}}$.

3. $\left\langle f_{1}, f_{2}\right\rangle_{L^{2}}=\int_{0}^{1}\left(f_{1} \odot f_{2}\right)=\int_{0}^{1}\left(f_{2} \odot f_{1}\right)=\left\langle f_{2}, f_{1}\right\rangle_{L^{2}}$.

4. Since $\left\langle f_{1}, f_{1}\right\rangle_{L^{2}}=\left\|f_{1}\right\|^{2}=D^{2}\left(f_{1}, 0\right)>0$, we have $\left\langle f_{1}, f_{1}\right\rangle_{L^{2}}>0$.

5. If $\left\langle f_{1}, f_{1}\right\rangle_{L^{2}}>0$, then $\left[\left\langle f_{1}, f_{1}\right\rangle_{L^{2}}\right]_{\alpha}>0$. Therefore, we have $\left[\left\langle f_{1}, f_{1}\right\rangle_{\alpha}^{-},\left\langle f_{1}, f_{1}\right\rangle_{\alpha}^{+}\right]>0$ and $\inf \left\langle f_{1}, f_{1}\right\rangle_{\alpha}^{-}>0$.

6. Since $\left\langle f_{1}, f_{1}\right\rangle_{L^{2}}=0$, we have $\left\|f_{1}\right\|^{2}=0$ and $D^{2}\left(f_{1}, 0\right)=0$. Hence, $f_{1} \ominus_{\mathrm{gH}} 0=0$. It follows that $f_{1}=0$.

Conversely, if $f_{1}=0$, then $f_{1} \ominus_{\mathrm{gH}} 0=0$. Thus, $\left\|f_{1} \ominus_{\mathrm{gH}} 0\right\|=0$. Since $D^{2}\left(f_{1}, 0\right)=0$, we have $\left\langle f_{1}, f_{1}\right\rangle_{L^{2}}=0$. Corollary 3.7 The vector space $F(\mathbb{R}, F(\mathbb{R}))$ with a FIP in the form of $\left\langle f_{1}, f_{2}\right\rangle_{L^{2}}=\int_{0}^{1} f_{1}(x) \odot f_{2}(x) d x$ is called a fuzzy inner product space (FIPS for short).

Lemma 3.8 Suppose that $F(\mathbb{R}, F(\mathbb{R}))$ is a vector space over $\mathbb{R}$. Also, the FIP in the fuzzy space $W^{1}[0,1]$ on $F(\mathbb{R}, F(\mathbb{R}))$ is a mapping as $\langle. \text {. . }\rangle_{1}: F(\mathbb{R}, F(\mathbb{R})) \times F(\mathbb{R}, F(\mathbb{R})) \rightarrow F(\mathbb{R})$, with this property for each $k \in F(\mathbb{R})$ and all functions $f_{1}, f_{2}, f_{3} \in F(\mathbb{R}, F(\mathbb{R}))$, satisfies the following conditions:

1. $\left\langle f_{1}+f_{2}, f_{3}\right\rangle_{1}=\left\langle f_{1}, f_{3}\right\rangle_{1} \oplus\left\langle f_{2}, f_{3}\right\rangle_{1}$,

2. $\left\langle k f_{1}, f_{2}\right\rangle_{1}=k\left\langle f_{1}, f_{2}\right\rangle_{1}$,

3. $\left\langle f_{1}, f_{2}\right\rangle_{1}=\left\langle f_{2}, f_{1}\right\rangle_{1}$,

4. $\left\langle f_{1}, f_{1}\right\rangle_{1}>0$,

5. if $f_{1} \neq 0$, then $\inf _{\alpha \in(0,1]}\left\langle f_{1}, f_{1}\right\rangle_{\alpha}^{-}>0$,

6. $\left\langle f_{1}, f_{1}\right\rangle_{1}=0$ iff $f_{1}=0$.

Proof To prove, we need to apply the fuzzy integral concepts, the Hausdorff distance and the fuzzy norm.

1. $\left\langle f_{1} \oplus f_{2}, f_{3}\right\rangle_{1}=\left(f_{1}(0) \oplus f_{2}(0)\right) \odot f_{3}(0) \oplus \int_{0}^{1}\left(f_{1}^{\prime} \oplus f_{2}^{\prime}\right) \odot f_{3}^{\prime}=$ $f_{1}(0) \odot f_{3}(0) \oplus f_{2}(0) \odot f_{3}(0) \oplus \int_{0}^{1}\left(f_{1}^{\prime} \odot f_{3}^{\prime}\right) \oplus \int_{0}^{1}\left(f_{2}^{\prime} \odot f_{3}^{\prime}\right)=$ $\left\langle f_{1}, f_{3}\right\rangle_{1} \oplus\left\langle f_{2}, f_{3}\right\rangle_{1}$.

2. $\left\langle k \odot f_{1}, f_{2}\right\rangle_{1}=k \odot f_{1}(0) \odot f_{2}(0) \oplus \int_{0}^{1}\left(k \odot f_{1}^{\prime} \odot f_{2}^{\prime}\right)=$ $k \odot f_{1}(0) \odot f_{2}(0) \oplus k \odot \int_{0}^{1}\left(f_{1}^{\prime} \odot f_{2}^{\prime}\right)=k \odot\left(f_{1}(0) \odot f_{2}(0)\right.$ $\left.\oplus \int_{0}^{1}\left(f_{1}^{\prime} \odot f_{2}^{\prime}\right)\right)=k \odot\left\langle f_{1}, f_{2}\right\rangle_{1}$.

3. $\left\langle f_{1}, f_{2}\right\rangle_{1}=f_{1}(0) \odot f_{2}(0) \oplus \int_{0}^{1}\left(f_{1}^{\prime} \odot f_{2}^{\prime}\right)=f_{2}(0) \odot f_{1}(0)$ $\oplus \int_{0}^{1}\left(f_{2}^{\prime} \odot f_{1}^{\prime}\right)=\left\langle f_{2}, f_{1}\right\rangle_{1}$.
The remaining proofs follow from the previous lemma.

Corollary 3.9 The vector space $F(\mathbb{R}, F(\mathbb{R}))$ with a FIP in the form $\left\langle f_{1}, f_{2}\right\rangle_{1}=f_{1}(0) \odot f_{2}(0) \oplus \int_{0}^{1} f_{1}^{\prime}(x) \odot f_{2}^{\prime}(x) d x$ is called a FIPS

In the following, assume that $\langle., .\rangle_{m}$ is the fuzzy inner product in the fuzzy space $W^{m}[0,1]$. Also, $\|$.$\| is the fuzzy$ norm.

Definition 3.10 A fuzzy sequence $\left\{\left(h_{n}, \alpha_{n}\right)\right\}$ in a FIPS $\left(W^{m}[0,1],\langle., .\rangle_{m}\right)$ is a fuzzy convergent if there is a fuzzy function $h \in W^{m}[0,1]$ such that $\lim _{n \rightarrow \infty}\left\|h_{n} \ominus_{\mathrm{gH}} h\right\|=0$ or $\lim _{n \rightarrow \infty} D\left(h_{n}, h\right)=0$, in which $\|h\|=\sqrt{\langle h, h\rangle}$.

Definition 3.11 A fuzzy sequence $\left\{\left(h_{n}, \alpha_{n}\right)\right\}$ in a FIPS $\left(W^{m}[0,1],\langle., .\rangle_{m}\right)$ is a fuzzy Cauchy if $\forall \varepsilon>0, \exists M>0: \forall m, n>M$, we have $\left\|h_{m} \ominus_{\mathrm{gH}} h_{n}\right\|<\varepsilon$, in which $\|h\|=\sqrt{\langle h, h\rangle}$.

Definition 3.12 The FIPS $\left(W^{m}[0,1],\langle., .\rangle_{m}\right)$ is a fuzzy complete if each fuzzy Cauchy sequence in $W^{m}[0,1]$ is a fuzzy convergent.

Definition 3.13 The FIPS is a fuzzy Hilbert space (FHS for short), while it is a fuzzy complete in the fuzzy norm $\|x\|=\sqrt{\langle x, x\rangle_{m}}$.

Corollary 3.14 The FIPS $\left(W^{m}[0,1],\langle., .\rangle_{m}\right)$ is a FHS, when it is a fuzzy complete in the fuzzy norm $\|\|=.\sqrt{\langle., .\rangle_{m}}$.

Definition 3.15 We consider $H$ as a FHS, with FIP $\left\langle f_{1}(x), f_{2}(x)\right\rangle_{H}$, in which $f_{1}(x)$ and $f_{2}(x)$ belong to $H$. If there is a fuzzy function such as $R_{y}(x) \in H$, where every $f_{1}(x)$ satisfies the condition $\left\langle f_{1}(x), R_{y}(x)\right\rangle_{H}=f_{1}(y)$, then $R_{y}(x)$ is called the FRK of $H$. Also, FHS of $H$ is called the FRKS.

\section{The exact and approximate solutions}

Definition 3.16 (Fuzzy Hilbert adjoint operator) Assume that $H_{1}$ and $H_{2}$ are fuzzy Hilbert spaces, and $L: H_{1} \rightarrow H_{2}$ is a fuzzy bounded linear operator, then $L^{*}: H_{2} \rightarrow H_{1}$ is the fuzzy Hilbert adjoint operator, while for each $x_{1} \in H_{1}$ and $x_{2} \in H_{2}$, we have $\left\langle L x_{1}, x_{2}\right\rangle_{H_{2}}=\left\langle x_{1}, L^{*} x_{2}\right\rangle_{H_{1}}$, where $\langle., .\rangle_{H_{i}}$ is the FIP in the FHS of $H_{i}$, in which $i=1,2$.

Definition 3.17 (Fuzzy Gram-Schmidt process) Given an arbitrary basis $\varphi_{1}, \varphi_{2}, \ldots, \varphi_{n}$ for a FIPS, if all the gH-differences are present, the fuzzy Gram-Schmidt process constructs by the fuzzy orthogonal basis $A_{1}, A_{2}, \ldots, A_{n}$ :

Step 1 Let $A_{1}=\varphi_{1}$,

Step 2 Let $A_{2}=\varphi_{2} \ominus_{\mathrm{gH}} \frac{\left\langle\varphi_{1}, \varphi_{2}\right\rangle}{\left\|\varphi_{1}\right\|^{2}} \varphi_{1}$, 


$$
\begin{aligned}
& \text { Step } 3 \text { Let } \quad A_{3}=\varphi_{3} \ominus_{\mathrm{gH}} \frac{\left\langle\varphi_{1}, \varphi_{3}\right\rangle}{\left\|\varphi_{1}\right\|^{2}} \varphi_{1} \ominus_{\mathrm{gH}} \\
& \frac{\left\langle\varphi_{2} \ominus_{\mathrm{gH}} \frac{\left\langle\varphi_{1}, \varphi_{2}\right\rangle}{\left\|\varphi_{1}\right\|^{2}} \varphi_{1}, \varphi_{3}\right\rangle}{\left\|\varphi_{2} \ominus_{\mathrm{gH}} \frac{\left\langle\varphi_{1}, \varphi_{2}\right\rangle}{\left\|\varphi_{1}\right\|^{2}} \varphi_{1}\right\|^{2}} \varphi_{2} \ominus_{\mathrm{gH}} \frac{\left\langle\varphi_{1}, \varphi_{2}\right\rangle}{\left\|\varphi_{1}\right\|^{2}} \varphi_{1}, \\
& \text { Step } n \quad \text { Let } A_{n}=\varphi_{n} \ominus_{\mathrm{gH}} \frac{\left\langle A_{1}, \varphi_{n}\right\rangle}{\left\|A_{1}\right\|^{2}} A_{1} \ominus_{\mathrm{gH}} \\
& \frac{\left\langle A_{2}, \varphi_{n}\right\rangle}{\left\|A_{2}\right\|^{2}} A_{2} \ldots \ominus_{\mathrm{gH}} \frac{\left\langle A_{n-1}, \varphi_{n}\right\rangle}{\left\|A_{n-1}\right\|^{2}} A_{n-1} \text {. }
\end{aligned}
$$

By normalizing $A_{1}, \ldots, A_{n}$ vectors, we can obtain the fuzzy normal orthogonal vectors of the form as follows:

$\overline{\varphi_{1}}=\frac{1}{\left\|A_{1}\right\|} A_{1}, \overline{\varphi_{2}}=\frac{1}{\| A_{2}} \| A_{2}, \ldots, \overline{\varphi_{n}}=\frac{1}{\left\|A_{n}\right\|} A_{n}$.

The fuzzy orthonormal function system $\left\{\overline{\varphi_{i}}(x)\right\}_{i=1}^{\infty}$ of the fuzzy space $W^{m}[0,1]$ will be obtained by fuzzy GramSchmidt orthogonalization process of $\left\{\varphi_{i}(x)\right\}_{i=1}^{\infty}$ as $\overline{\varphi_{i}}(x)=\sum_{k=1}^{i} \beta_{i k} \varphi_{k}(x)$, where $\beta_{i k}$ are orthogonalization coefficients given as $\beta_{11}=\frac{1}{\left\|\varphi_{1}\right\|}, \quad \beta_{i i}=\frac{1}{d_{i k}}$, and $\beta_{i j}=-\left(\frac{1}{d_{i k}}\right) \sum_{k=j}^{i-1} c_{i k} \beta_{k j} \quad$ for $\quad j<i, \quad$ while $d_{i k}=\sqrt{\left\|\varphi_{i}\right\|^{2}-\sum_{k=1}^{i-1} c_{i k}^{2}}, c_{i k}=\left\langle\varphi_{i}, \overline{\varphi_{k}}\right\rangle$, and $\left\{\varphi_{i}(x)\right\}_{i=1}^{\infty}$ is the orthonormal system in the space $W^{m}[0,1]$.

Definition 3.18 We consider $\left\{\overline{\varphi_{i}}(x)\right\}_{i=1}^{\infty}$ as a fuzzy orthonormal system, we have:

$\left\langle\overline{\varphi_{i}}(x), \overline{\varphi_{j}}(x)\right\rangle= \begin{cases}0 & i \neq j ; \\ 1 & i=j .\end{cases}$

where 0 and 1 are fuzzy numbers.

Assume that $L y(x)=y^{\prime \prime}(x)+m(x) y^{\prime}(x)+n(x) y(x)$ in the equation of (1.1). In this case, $L: W^{m}[0,1] \rightarrow W^{1}[0,1]$ is a fuzzy bounded linear operator. We take $\delta_{i}(x)=R_{x_{i}}(x)$ and $\varphi_{i}(x)=L^{*} \delta_{i}(x)$ where $R_{x_{i}}(x)$ is the FRK. Also, $L^{*}$ is the fuzzy adjoint operator of $L$. Using the concepts of the FRK, for each $y(x)$, the following equality is true:

$\left\langle y(x), \delta_{i}(x)\right\rangle=\left\langle y(x), R_{x_{i}}(x)\right\rangle=y\left(x_{i}\right)$.

Regarding the above and using the properties of $R_{x}(t)$, we get $\left\langle y(x), \varphi_{i}(x)\right\rangle=\left\langle y(x), L^{*} \delta_{i}(x)\right\rangle=\left\langle L y(x), \delta_{i}(x)\right\rangle=L y\left(x_{i}\right), \quad i \in \mathbb{N}$.

Moreover,

$\varphi_{i}(x)=\left\langle\varphi_{i}(t), R_{x}(t)\right\rangle=\left\langle L^{*} \delta_{i}(t)\right.$,

$\left.R_{x}(t)\right\rangle=\left\langle\delta_{i}(t), L_{t} R_{x}(t)\right\rangle=\left.L_{t} R_{x}(t)\right|_{t=x_{i}}$.
In this case, $\varphi_{i}(x)=\left.L_{t} R_{x}(t)\right|_{t=x_{i}}$, where $L_{t}$ is the fuzzy operator $L$ that applies to the function of $t$.

By the fuzzy Gram-Schmidt process, we orthonormalize the sequence $\left\{\varphi_{i}(x)\right\}_{i=1}^{\infty}$ and we get the fuzzy orthonormal system $\left\{\overline{\varphi_{i}}(x)\right\}_{i=1}^{\infty}$, that is, $\overline{\varphi_{i}}(x)=\sum_{k=1}^{i} \beta_{i k} \varphi_{k}(x), \quad\left(\beta_{i i}>0, i=1,2, \ldots\right)$, where $\beta_{i k}$ are coefficients of Gram-Schmidt orthonormalization and $\left\{\overline{\varphi_{i}}(x)\right\}_{i=1}^{\infty}$ is a fuzzy orthonormal basis of the fuzzy space $W^{m}[0,1]$.

Theorem 3.19 Assume that the solution of equation of (1.1) is unique. The exact solution of this equation is as $y(x)=\sum_{i=1}^{\infty} \sum_{k=1}^{i} \beta_{i k} f\left(x_{k}\right) \overline{\varphi_{i}}(x)$.

Proof Suppose that $y(x)$ is the solution of equation of (1.1) given that $\left\{\overline{\varphi_{i}}(x)\right\}_{i=1}^{\infty}$ is a fuzzy orthonormal system. In this case, the following equalities are true:

$$
\begin{aligned}
y(x) & =\sum_{i=1}^{\infty}\left\langle y(x), \bar{\varphi}_{i}(x)\right\rangle \overline{\varphi_{i}}(x) \\
& =\sum_{i=1}^{\infty}\left\langle y(x), \sum_{k=1}^{i} \beta_{i k} \varphi_{k}(x)\right\rangle \overline{\varphi_{i}}(x) \\
& =\sum_{i=1}^{\infty} \sum_{k=1}^{i} \beta_{i k}\left\langle y(x), \varphi_{k}(x)\right\rangle \overline{\varphi_{i}}(x) \\
& =\sum_{i=1}^{\infty} \sum_{k=1}^{i} \beta_{i k}\left\langle y(x), L^{*} \delta_{k}(x)\right\rangle \overline{\varphi_{i}}(x) \\
& =\sum_{i=1}^{\infty} \sum_{k=1}^{i} \beta_{i k}\left\langle L y(x), \delta_{k}(x)\right\rangle \overline{\varphi_{i}}(x) \\
& =\sum_{i=1}^{\infty} \sum_{k=1}^{i} \beta_{i k}\left\langle L y(x), R_{x_{k}}(x)\right\rangle \overline{\varphi_{i}}(x) \\
& =\sum_{i=1}^{\infty} \sum_{k=1}^{i} \beta_{i k} L y\left(x_{k}\right) \overline{\varphi_{i}}(x) \\
& =\sum_{i=1}^{\infty} \sum_{k=1}^{i} \beta_{i k} f\left(x_{k}\right) \overline{\varphi_{i}}(x),
\end{aligned}
$$

where $\langle.,$.$\rangle is the fuzzy inner product. Also, the approximate$ solution of (1.1) is as

$y_{n}(x)=\sum_{i=1}^{n} \sum_{k=1}^{i} \beta_{i k} f\left(x_{k}\right) \overline{\varphi_{i}}(x)$. 


\section{Fuzzy convergence analysis}

Here, we examine the fuzzy convergence.

Lemma 4.1 Let $\left(W^{m}[0,1],\langle., .\rangle_{m}\right)$ be a FIPS. Also, $y(x) \in W^{m}[0,1]$. In this case, there is a constant $n$ such that

1. $|y(x)| \leq n\|y(x)\|_{m}$

2. $\left|y_{\mathrm{gH}}^{(k)}(x)\right| \leq n\|y(x)\|_{m}, \quad(k=1,2, \ldots, m-1)$.

Proof Using the property of the FRK in the Definition 3.15, we have $y(x)=\left\langle y(.), R_{x}(.)\right\rangle_{m}$. Also, by Lemma 3.2 in [8], we can conclude that $|y(x)|=\left|\left\langle y(.), R_{x}(.)\right\rangle_{m}\right| \leq\|y(.)\|_{m}\left\|R_{x}(.)\right\|_{m}$. Therefore, there is a constant $n$ such that $|y(x)| \leq n\|y\|_{m}$.

Also, $y_{\mathrm{gH}}^{(k)}(x)=\left\langle y(.), R_{x}^{(k)}(.)\right\rangle_{m}$. In this case,

$$
\begin{aligned}
& \left|y_{\mathrm{gH}}^{(k)}(x)\right|=\left|\left\langle y(.), R_{x}^{(k)}(.)\right\rangle_{m}\right| \\
& \quad \leq\|y(.)\|_{m}\left\|R_{x}^{(k)}(.)\right\|_{m} \leq n_{k}\|y\|_{m}, \\
& \quad(k=1, \ldots, m-1) .
\end{aligned}
$$

Hence, it is enough to suppose $n=\max _{1 \leq k \leq m-1}\left\{n_{k}\right\}$.

Corollary 4.2 By Definition 3.10, a fuzzy sequence $\left\{\left(y_{n}, \alpha_{n}\right)\right\}$ in a FIPS $\left(W^{m}[0,1],\langle., .\rangle_{m}\right)$ is called a fuzzy convergent when there is a fuzzy function $y_{n}$ in the fuzzy space $W^{m}[0,1]$ such that

$\lim _{n \rightarrow \infty}\left\|y_{n} \ominus_{\mathrm{gH}} y\right\|=0$.

where $y$ is an exact solution and $y_{n}$ is an approximate solution of (1.1).

Theorem 4.3 The approximate solution $y_{n}(x)$ is uniformly fuzzy convergent. Also, for all $k=1,2, \ldots, m-1$, the derivatives $y_{n}^{(k)}(x)$ are all uniformly fuzzy convergent.

Proof If $y(x)$ be solution of equation of (1.1) in the fuzzy space $W^{m}[0,1]$, then we have:

$$
\begin{aligned}
\left|y_{n}(x) \ominus_{\mathrm{gH}} y(x)\right| & =\left|\left\langle y_{n}(.) \ominus_{\mathrm{gH}} y(.), R_{x}(.)\right\rangle\right| \\
& \leq\left\|y_{n}(.) \ominus_{\mathrm{gH}} y(.)\right\|\left\|R_{x}(.)\right\| \\
& \leq M\left\|y_{n}(.) \ominus_{\mathrm{gH}} y(.)\right\|
\end{aligned}
$$

and

$$
\begin{aligned}
& \left|y_{n}^{(k)}(x) \ominus_{\mathrm{gH}} y_{\mathrm{gH}}^{(k)}(x)\right|=\left|\left\langle y_{n}(.) \ominus_{\mathrm{gH}} y(.), R_{x}^{(k)}(.)\right\rangle\right| \\
& \quad \leq\left\|y_{n}(.) \ominus_{\mathrm{gH}} y(.)\right\|\left\|R_{x}^{(k)}(.)\right\| \\
& \quad \leq N_{k}\left\|y_{n}(.) \ominus_{\mathrm{gH}} y(.)\right\|,(k=1,2, \ldots, m-1) .
\end{aligned}
$$

Hence, it is enough to suppose $N=\max _{1 \leq k \leq m-1}\left\{N_{k}\right\}$ where $M, N>0$ are constants. If $\lim _{n \rightarrow \infty}\left\|y_{n}(.) \ominus_{\mathrm{gH}} y().\right\|=0$, then the approximate solution $y_{n}(x)$ (resp. $\left.y_{n}^{(k)}(x)\right)$ is uniformly fuzzy convergent to the exact solution $y(x)\left(\right.$ resp. $y_{\mathrm{gH}}^{(k)}(x)$ ).

To solve examples of "Examples" section, by [6], we need to define the space $W^{3}[0,1]$ and the inner product in this space as $W^{3}[0,1]=\left\{y(x) \mid y^{\prime \prime}\right.$ is an absolutely continuous function, $\left.y^{\prime \prime \prime}(x) \in L^{2}[0,1], y(0)=0, y(1)=0\right\}$.

$$
\begin{gathered}
\langle y, z\rangle=y(0) z(0)+y^{\prime}(0) z^{\prime}(0)+y^{\prime \prime}(0) z^{\prime \prime}(0) \\
+\int_{0}^{1} y^{\prime \prime \prime}(x) z^{\prime \prime \prime}(x) \mathrm{d} x ; y, z \in W^{3}[0,1] .
\end{gathered}
$$

In this case, reproducing kernel $R_{v}(u)$ is as

$R_{v}(u)= \begin{cases}l R_{v}(u)=\sum_{i=1}^{6} c_{i}(v) u^{i-1}, & u<v, \\ r R_{v}(u)=\sum_{i=1}^{6} d_{i}(v) u^{i-1}, & u \geqslant v .\end{cases}$

where $c_{i}(v)$ and $d_{i}(v)$ are obtained by the following relationships:

$\frac{\partial^{i} l R_{v}(v)}{\partial u^{i}}=\frac{\partial^{i} r R_{v}(v)}{\partial u^{i}}, \quad i=0,1, \ldots, 4 ;$

$(-1)^{3}\left(\frac{\partial^{5} R_{v}\left(v^{+}\right)}{\partial u^{5}}-\frac{\partial^{5} R_{v}\left(v^{-}\right)}{\partial u^{5}}\right)=1$,

and

$$
\begin{cases}\frac{\partial^{i} R_{v}(0)}{\partial u^{i}}-(-1)^{2-i} \frac{\partial^{5-i} R_{v}(0)}{\partial u^{5-i}}=0, & i=1,2 \\ \frac{\partial^{5-i} R_{v}(1)}{\partial u^{5-i}}=0, & i=1,2 \\ R_{v}(0)=0 & \\ R_{v}(1)=0 & \end{cases}
$$


Thus, the representation of the reproducing kernel in $W^{3}[0,1]$ is as

Example 5.2 Suppose the equation of boundary value is as

$R_{v}(u)= \begin{cases}\frac{1}{48} u(-1+v)\left(6(-2+v) v+3 u(-2+v) v-u^{2}(-4+v)(2+v)\right), & u<v ; \\ -\frac{1}{48}(-1+u) v\left(-8 v^{2}-2 u\left(-6-3 v+v^{2}\right)+u^{2}\left(-6-3 v+v^{2}\right)\right), & u \geqslant v .\end{cases}$

Therefore,

$R_{v}(u)= \begin{cases}\frac{1}{48} u(-1+v)\left(-12 v+6 v^{2}-6 u v+3 u v^{2}+8 u^{2}+2 u^{2} v-u^{2} v^{2}\right), & u<v ; \\ \frac{1}{48} v(-1+u)\left(8 v^{2}-12 u-6 u v+2 u v^{2}+6 u^{2}+3 u^{2} v-u^{2} v^{2}\right), & u \geqslant v .\end{cases}$

\section{Examples}

Here are some examples to better understand. In the following examples, taking into account the reproducing kernel space $W^{m}[a, b]$ and the reproducing kernel in this space and applying the reproducing kernel method, the exact solution of the equation is obtained. Also, we can obtain the approximate solution of this equation using the exact solution. Using the method presented in this paper (i.e, reproducing kernel method), taking:

$x_{i}=a+(i-1) d x, \quad d x=\frac{b-a}{n-1}, \quad i=1,2, \ldots, n$.

we can simply discuss about $\left|y(x)-y_{n}(x)\right|$, for every positive $n$, in the different reproducing kernel spaces. This process is similar to [12].

Example 5.1 We consider the following boundary value problem. Then, we find its exact solution.

$\left\{\begin{array}{l}y^{\prime \prime}(x)+200 \mathrm{e}^{x} y^{\prime}(x)+300 \sin (x) y(x)=f(x), \quad 0<x<1, \\ y(0)=0, \quad y(1)=0,\end{array}\right.$

where $f(x)=\sinh (x)+200 \mathrm{e}^{x}(\cosh (x)-\sinh (1))+300 \sin (x)$ $(\sinh (x)-x \sinh (1))$. The exact solution is given by $y(x)=\sinh (x)-x \sinh (1)$.

$$
\left\{\begin{array}{l}
y^{\prime \prime}(x)+200 \mathrm{e}^{x} y^{\prime}(x)+300 \sin (x) y(x)=f(x), \quad 0<x<1 \\
y(0)=0, \quad y(1)=0
\end{array}\right.
$$

where $f(x)=2 \sinh (x)+x \cosh (x)+200 \mathrm{e}^{x}(\cosh (x)+x \sinh (x)-$ $\cosh (1))+300 \sin (x)(x \cosh (x)-x \cosh (1))$. Then, we simply see that the exact solution is as $y(x)=x \cosh (x)-x \cosh (1)$.

Example 5.3 If the boundary value problem is as

$\left\{\begin{array}{l}y^{\prime \prime}(x)+200 \mathrm{e}^{x} y^{\prime}(x)+300 \cos (x) y(x)=f(x), \quad 0<x<1, \\ y(0)=0, \quad y(1)=0,\end{array}\right.$

where $f(x)=\sinh (x)+200 \mathrm{e}^{x}(\cosh (x)-\sinh (1))+300 \cos$ $(x)(\sinh (x)-x \sinh (1))$, then the exact solution is as $y(x)=\sinh (x)-x \sinh (1)$.

\section{Conclusion}

In this paper, the definitions of fuzzy Cauchy, fuzzy complete and fuzzy inner product were studied. Moreover, we presented the solution of fuzzy second-order two-point boundary value problem by the fuzzy reproducing kernel method. We had a lot of limitations compared to the real state, and all the lemmas and the theorems were not easily verifiable. The basis for what we did was based on the existence of the gH-differences.

Open Access This article is distributed under the terms of the Creative Commons Attribution 4.0 International License (http://creativeco 
mmons.org/licenses/by/4.0/), which permits unrestricted use, distribution, and reproduction in any medium, provided you give appropriate credit to the original author(s) and the source, provide a link to the Creative Commons license, and indicate if changes were made.

\section{References}

1. Arqub, O.A.: The reproducing kernel algorithm for handing differential algebraic systems of ordinary differential equations. Math. Methods Appl. Sci. 39, 4549-4562 (2016)

2. Arqub, O.A., AL-Smadi, M., Momani, Sh, Hayat, T.: Application of reproducing kernel algorithm for solving second-order, two-point fuzzy boundary value problems. Soft Comput. 21, 7191-7206 (2017)

3. Arqub, O.A., AL-Smadi, M., Momani, Sh., Hayat, T.: Numerical solutions of Fuzzy differential equations using reproducing kernel Hilbert space method. Soft Comput. 20, 3283-3302 (2016)

4. Allahviranloo, T., Gouyandeh, Z., Armand, A.: A full Fuzzy method for solving differential equation based on Taylor expansion. Journal of Intelligent \& Fuzzy Systems 29, 1039-1055 (2015)

5. Bede, B., Stefanini, L.: Generalized differentiability of fuzzyvalued functions. Fuzzy Sets Syst. 230, 119-141 (2013)
6. Cui, M., Lin, Y.: Nonlinear Numerical Analysis in the Reproducing Kernel Space. Nova Science Pub. Inc., Hauppange (2009)

7. Guang-Quan, Z.: Fuzzy continuous function and its properties. Fuzzy Sets Syst. 43(2), 159-171 (1991)

8. Hasankhani, A., Nazari, A., Saheli, M.: Some properties of fuzzy Hilbert spaces and norm of operators. Iran. J. Fuzzy Syst. 7(3), 129-157 (2010)

9. Kaleva, O.: Fuzzy differential equations. Fuzzy Sets Syst. 24, 301-317 (1987)

10. Khalilpour, K., Allahviranloo, T.: An initial-value method for twopoint fuzzy boundary value problems. World Appl. Sci. J. 13(10), 2148-2155 (2011)

11. Kider, J.R., Sabre, R.I.: Fuzzy Hilbert spaces. Eng. Tech. J. 28(9), 1816-1824 (2010)

12. Li, X.Y., Wu, B.Y.: Error estimation for the reproducing kernel method to solve linear boundary value problems. J. Comput. Appl. Math. 243, 10-15 (2013)

13. Negoita, C.V., Ralescu, D.: Applications of Fuzzy Sets to Systems Analysis. Wiley, New York (1975)

14. Stefanini, L., Bede, B.: Generalized Hukuhara differentiability of interval-valued functions and interval differential equations. Nonlinear Anal. 71, 1311-1328 (2009)

Publisher's Note Springer Nature remains neutral with regard to jurisdictional claims in published maps and institutional affiliations. 